\title{
Modeling glucose and free fatty acid kinetics in glucose and meal tolerance test
}

\author{
Yanjun Li ${ }^{1}$, Carson C. Chow ${ }^{1}$, Amber B. Courville ${ }^{2}$, Anne E. Sumner ${ }^{3}$ and Vipul Periwal ${ }^{1 *}$
}

\author{
* Correspondence: \\ vipulp@mail.nih.gov \\ 'Laboratory of Biological Modeling, \\ National Institute of Diabetes and \\ Digestive and Kidney Diseases \\ (NIDDK), National Institutes of \\ Health (NIH), MSC 5621, LBM, \\ NIDDK, NIH, Bethesda, MD \\ 20892-5621, USA \\ Full list of author information is \\ available at the end of the article
}

\begin{abstract}
Background: Quantitative evaluation of insulin regulation on plasma glucose and free fatty acid (FFA) in response to external glucose challenge is clinically important to assess the development of insulin resistance (World J Diabetes 1:36-47, 2010). Mathematical minimal models (MMs) based on insulin modified frequently-sampled intravenous glucose tolerance tests (IM-FSIGT) are widely applied to ascertain an insulin sensitivity index (IEEE Rev Biomed Eng 2:54-96, 2009). Furthermore, it is important to investigate insulin regulation on glucose and FFA in postprandial state as a normal physiological condition. A simple way to calculate the appearance rate (Ra) of glucose and FFA would be especially helpful to evaluate glucose and FFA kinetics for clinical applications.
\end{abstract}

Methods: A new MM is developed to simulate the insulin modulation of plasma glucose and FFA, combining IM-FSIGT with a mixed meal tolerance test (MT). A novel simple functional form for the appearance rate (Ra) of glucose or FFA in the MT is developed. Model results are compared with two other models for data obtained from 28 non-diabetic women (13 African American, 15 white).

Results: The new functional form for Ra of glucose is an acceptable empirical approximation to the experimental Ra for a subset of individuals. When both glucose and FFA are included in FSIGT and MT, the new model is preferred using the Bayes Information Criterion (BIC).

Conclusions: Model simulations show that the new MM allows consistent application to both IM-FSIGT and MT data, balancing model complexity and data fitting. While the appearance of glucose in the circulation has an important effect on FFA kinetics in MT, the rate of appearance of FFA can be neglected for the time-period modeled.

Keywords: Glucose tolerance test, Lipolysis, Appearance rate

\section{Background}

Insulin is one of the primary factors regulating plasma glucose and free fatty acid (FFA). The development of insulin resistance and type 2 diabetes (T2D) is closely associated with the abnormal regulation of insulin on carbohydrate metabolism. Dysfunction of whole body lipid metabolism plays an important role in the development of T2D [1-4]. The modulation of glucose and FFA metabolism by insulin depends on race, obesity, sex, and regional body fat distribution [5-7]. Quantitation of insulin regulation of glucose and FFA, including kinetic interactions in response to external physiological stimuli, is clinically important because of the potential to inform treatment paradigms for T2D.

(c) $2016 \mathrm{Li}$ et al. Open Access This article is distributed under the terms of the Creative Commons Attribution 4.0 International License (http://creativecommons.org/licenses/by/4.0/), which permits unrestricted use, distribution, and reproduction in any medium, provided you give appropriate credit to the original author(s) and the source, provide a link to the Creative Commons license, and indicate if changes were made. The Creative Commons Public Domain Dedication waiver (http://creativecommons.org/ publicdomain/zero/1.0/) applies to the data made available in this article, unless otherwise stated. 
Mathematical indexes have been defined to assess the effects of insulin on carbohydrate metabolism [8]. The insulin sensitivity index $\left(S_{I}\right)$ is the most frequently used, as directly measured by glucose clamp [9], or indirectly by a mathematical MM analysis of data from the insulin-modified frequently-sampled intravenous glucose tolerance test (IM-FSIGT) [10-13]. In addition, the FSIGT MM assesses the dynamic relationship between insulin and glucose, and is used clinically.

This approach has well-known limitations [8]. First and foremost, most MMs consider only insulin and glucose $[14,15]$. Impaired lipid metabolism is related to the development of insulin resistance [16], so the regulation of lipid metabolism by insulin must be assessed. While some MMs have investigated lipid dynamics [17-19], parameters in these models are often based on mechanisms with scant physiological support. Neither glucose clamp nor FSIGT is a "normal" state, which limits physiological insights from these approaches. Therefore, we aim to quantify whole body regulation by insulin of carbohydrate and lipid metabolism simultaneously in response to meals. Secondly, the insulin-glucose system is obviously connected so using measured insulin as an external input in the model is conceptually suspect. The insulin-modified FSIGT does have an administered bolus of exogenous insulin to assess insulin sensitivity independent of endogenous insulin secretion through the MM parameters. Nevertheless, an ideal model would be autonomic, and starting from an initial measurement of insulin and glucose, would predict the subsequent time-course of both variables, given subject-specific parameters for such a model. However, such an autonomic model would also apply only to a state with no meals ingested. Clearly, this would not address the former limitation of the MM approach.

In the postprandial state, dietary glucose and FFA enters the circulation after digestion and absorption [20,21]. Their rates of appearance ( $\mathrm{Ra}$ ) have to be taken into account. Although Ra can be measured by isotope tracer technique [22], this is expensive, not always feasible, and typically provides sparse data. Mathematical approaches have been applied to evaluate glucose and FFA kinetics in the postprandial state. Some studies modeled glucose responses in oral glucose tolerance test (OGTT) or meal test (MT) [23-27]. The Ra of glucose was introduced with a complex algorithm and many parameters, limiting clinical utility. Some models for postprandial FFA metabolism [28, 29], however, did not consider Ra of FFA.

Indeed, the FSIGT and the mixed meal models are trying to fit complex physiological processes with simplified models involving just a few parameters. For example, the FSIGT ignores hepatic glucose output as an independent process. The aim, of course, is to ascribe some overall biological relevance to these few parameters, as has been done for decades with the insulin sensitivity index. Our aim in this paper is to figure out if a simple Ra function can actually model the complex physiology of food ingestion and absorption through the gastro-intestinal tract, when used along with the intravenous glucose model. The point is that if the Ra combined with the intravenous glucose model is inadequate for the MT, this inadequacy will show up as an additional contribution to the Ra of glucose, and hence as a bad fit with the functional parameterization of the Ra. A comparison of the adequacy of this Ra functional parameterization for two ethnic groups is a test of the general applicability of this simplified physiology in this controlled setting. 
The specific aims of our study are: first, to develop a new MM to evaluate insulin regulation of glucose and FFA in both IM-FSIGT and MT together; and second, to develop a simplified empirical function to parametrize the Ra of glucose or FFA in MT so that contributions of $\mathrm{Ra}$ on glucose or FFA responses can be quantitatively evaluated with no change in the equations from the FSIGT equations. This may be helpful to evaluate Ra of glucose or FFA in a postprandial state clinically as a simpler, albeit limited, alternative to tracer studies.

The model augments a previously developed model of the response of FFA and glucose to insulin in IM-FSIGT alone [30]. Experimental data from two ethnic groups African American women (AA) and white women are studied. The contributions of Ra of glucose and FFA on corresponding glucose or FFA kinetics in MT are evaluated with different definitions of $\mathrm{Ra}$ in various simulation combinations, and compared with another two published models. The parameter values for each group obtained in each model are determined and compared.

\section{Methods}

\section{Subjects}

Premenopausal women - 13 African-Americans (AA) and 15 white matched for age and body mass index (BMI) participated (Additional file 1: Table S1). Recruitment was by flyers, newspaper advertisements, and the National Institutes of Health web site. Only nondiabetic women with normal hemograms, liver, kidney, and thyroid function were enrolled. Participants were not taking medications known to affect either glucose or lipid metabolism, or oral contraceptives. Because the menstrual cycle does not impact FFA metabolism, studies were performed throughout the cycle [7, 31]. The Institutional Review Board of the National Institute of Diabetes and Digestive and Kidney Diseases approved the study. All subjects gave informed consent.

\section{Experimental protocol and measurement IM-FSIGT}

The experimental design of IM-FSIGT is as in previous work [5, 7, 30]. Each subject underwent an insulin modified frequently sampled intravenous glucose tolerance test (IM-FSIGT). The IM-FSIGT was performed in the morning after a 12-h overnight fast. Intravenous catheters were placed in both antecubital veins. Arterialized blood samples were collected for analysis. At time 0 , dextrose $(0.3 \mathrm{~g} / \mathrm{kg})$ was injected over $1 \mathrm{~min}$ and a bolus injection of insulin $(0.03 \mathrm{U} / \mathrm{kg})$ was given at $20 \mathrm{~min}$. Samples for glucose, insulin, and FFA were obtained at $-10,-1,0,1,2,3,4,5,6,7,8,10,12,14,16,20,22,23,24$, $25,27,30,40,50,60,70,80,90,100,120,150$, and $180 \mathrm{~min}$.

\section{MT}

The experimental design of meal test (MT) has been described in detail previously [32]. After a 7-day diet equilibration period [32], in which all of subjects' meals were provided through a metabolic kitchen, subjects were provided with standardized breakfast. The standardized breakfast consisted of $30 \%$ of the participants' energy needs on the last day of the equilibration period, $20 \%$ protein, $40 \%$ fat and $40 \%$ carbohydrate. Plasma samples for glucose, insulin and FFA were obtained at 0, 30, 60, 90, 120, 150, 180, 210, 240, 270, 300, 330 and $360 \mathrm{~min}$. Triglyceride (TG) were also measured at 0, 120, 240, $360 \mathrm{~min}$. 
Glucose and TG were measured on Dimension Vista 1500 analyzers (Siemens) using standard automated methods. Insulin was measured using an immunochemiluminometric assay from Diagnostic Products on an Immulite 2500 machine (Diagnostic Products). FFA was measured with Wako HR Series NEFA-HR kit (Wako Diagnostics, Wako Chemicals USA, Inc., Richmond, VA) and run on a COBAS FARA-II analyzer (Roche Diagnostics, Indianapolis, IN). Physiological variables associated with anthropometrics (e.g., waist, hip, and thigh circumferences), VAT and SAT, body composition etc. were measured as in previous studies [7].

Insulin resistance represented by insulin sensitivity index $\left(\mathrm{S}_{\mathrm{I}}\right)$ was determined from the MM, given below. The acute insulin responses to glucose (AIRg) in IMFSIGT was calculated as the incremental area under the curve (AUC) for insulin between 0 and $10 \mathrm{~min}$ for the insulin concentration above basal level. The disposition index (DI), calculated as the product of AIRg of IM-FSIGT and $S_{\mathrm{I}}$, was determined as a measure of $\beta$-cell function, specifically defined as the ability of the circulating insulin concentration to compensate for insulin resistance. Statistical analysis was performed with Statistica (www.statsoft.com). The null hypothesis is there is no difference between AA women and white women. The critical value is $\alpha=0.05$.

\section{Model description}

\section{Model structure}

The new model (MOD 1, detailed in Additional file 1, Section B) is partly based on our previous study [30]. Briefly, the main modification is that 1) in the dynamic equation of plasma FFA, it is assumed that insulin can stimulate the FFA clearance [33-37] as well; 2) rate of appearance ( $\mathrm{Ra}$ ) terms are introduced in the dynamic equations for glucose or FFA in meal test (MT), representing the net appearance of glucose or FFA into circulation via digestive system after a meal.

To thoroughly investigate the performance of the present model and the influence of Ra on glucose or FFA dynamics in MT, this model was compared with two published models -MOD 2 [30] and MOD 3 [29]. In brief, MOD 1 and MOD 2 have the same dynamic mass balance equation for glucose and insulin kinetics. They are distinguished in FFA clearance. FFA kinetics equation has two terms lipolysis and clearance. MOD 2 assumes the constant clearance rate. MOD 1 assumes clearance is insulin-dependent. Both MOD 1 and MOD 2 assume that insulin modulates glucose and FFA via the same insulin action $(X$, or remote insulin compartment). MOD 3 directly uses insulin with specific time delay for lipolysis and clearance, respectively. The appearance rate (Ra) of glucose or FFA in MT is introduced under specific simulation combinations. The components of the three models are compared in Table 1. The descriptions of all parameters are listed in Additional file 1: Table S2.

\section{Appearance rate of glucose and FFA}

To avoid too many parameters and complex algorithms, empirical functional forms were used to simplify the expression and simulation of Ra. According to experimental measurement [38,39] and other mathematical studies [23, 27], two different equations for Ra were used. 
Table 1 Comparison of each component of three models

\begin{tabular}{|c|c|c|c|}
\hline & MOD 1 & MOD 2 & MOD 3 \\
\hline Glucose kinetics & $S_{G} G_{b}-\left(S_{G}+S_{1} X\right) G$ & & - \\
\hline Insulin kinetics & $C_{x}\left[/(t)-X-I_{b x}\right]$ & & - \\
\hline FFA lipolysis & $10+\frac{1_{2}}{1+\left(X / X_{2}\right)^{A_{\text {I. }} 0}}$ & $I_{0}+\left[\frac{I_{2}}{1+\left(X / X_{2}\right)^{A_{1}}}\right]$ & $\frac{V_{m}^{L i p}}{1+\left(I\left(t-t_{\text {Delaylip }}\right) / K_{\text {Lip }}\right)^{h_{\text {hip }}}}$ \\
\hline FFA clearance & $C_{f}\left[1+\frac{\left(X / K_{C l}\right)^{A_{C l}}}{1+\left(X / K_{C l}\right)^{A_{C l}}}\right] F$ & $C_{f 0} F$ & 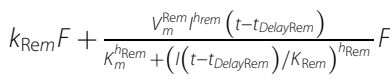 \\
\hline
\end{tabular}

$G$ is plasma glucose, $F$ is plasma FFA, $I$ is plasma insulin as model input, $X$ is the insulin action representing the indirect effect of insulin on the regulation of plasma glucose and FFA. The detailed descriptions of parameters are listed in Additional file 1: Table S2. Note that $I_{b x}$ is a fitted parameter that is determined through the action of $X$ on glucose and FFA. Thus, it is not necessarily the same as measured basal insulin, and we have used the subscript $b x$ to emphasize this fact

Type I is similar to a log-normal function:

$$
\begin{aligned}
& R a_{G}(t)= \begin{cases}0 \\
\frac{\Delta_{G}}{t \sigma_{G}} \exp \left\{-\left[\frac{\left[\ln \left(t / m_{G}\right)\right]^{2}}{2 \sigma_{G}^{2}}\right]\right\} & \mathrm{t}=0 \\
\text { otherwise }\end{cases} \\
& R a_{F}(t)= \begin{cases}0 & t \leq t_{0 F} \\
\frac{\Delta_{F}}{\left(t-t_{0 F}\right) \sigma_{F}} \exp \left(-\left[\frac{\left[\ln \left(\left(t-t_{0 F}\right) / m_{F}\right)\right]^{2}}{2 \sigma_{F}^{2}}\right]\right) & \text { otherwise }\end{cases}
\end{aligned}
$$

where $\Delta_{G}, \Delta_{F}$ are phenomenological magnitude factors, $\mathrm{m}_{\mathrm{G}}, \mathrm{m}_{\mathrm{F}}$ are time scale parameters, $\sigma_{F}$ and $\sigma_{G}$ are width parameters, and $\mathrm{t}_{0 \mathrm{~F}}$ is the specific time at which FFA originating from chylomicron triglyceride appears in the plasma. The areas under the curves (AUC) of glucose and FFA Ra for different periods of MT were calculated. Two variables are fixed $t_{0 F}=60 ; m_{F}=300$ based on a preliminary study.

Type II is from Pearson et al.'s study [40],

$$
R a_{G}(t)=\frac{\phi_{G} t}{\tau_{G}^{2}} \exp \left\{-t^{2} /\left(2 \tau_{G}^{2}\right)\right\}
$$

in which $\phi_{G}$ is the magnitude coefficient, and $\tau_{G}$ is the timescale coefficient. Both of them have to be determined, as compared to three parameters that need to be determined for the Type I glucose Ra.

\section{Simulation combinations}

The behaviors of the three models were compared under four simulation Combinations: 1) without considering Ra for glucose and FFA; 2) considering Ra of glucose and FFA expressed as Type I functions; 3 ) only Ra of glucose is incorporated expressed as a Type I function; 4) only Ra of glucose is incorporated, expressed as a Type II function [40]. While it seems obvious that excluding Ra for glucose and FFA (Combination 1 above) is unnecessary, we present it for completeness so that the difference between Combination 2 and Combination 3 can be clearly exhibited in the progression: no Ra, both Ra's, only glucose Ra. To completely investigate the parameter stability, based on these results, additional combinations are involved for MOD 1 and MOD 2, S1) only IM-FSIGT; S2) only MT (Type I function); S3) only MT(Type II function). With experiment data from IM-FSIGT and MT in each subject, the individual parameters are determined for each model and each Combination, respectively. The simulations of glucose and FFA dynamics of each model and each Combination 
can then be compared. Moreover, Bayes information criterion (BIC) was applied to evaluate the simulation results of three models with each combination.

\section{Calculation of $R a$}

Herrero et al.'s study [25] provided an alternative way to evaluate Ra needing somewhat more involved calculations. According to the glucose dynamic equation, $\mathrm{Ra}$ is function of glucose derivative and glucose concentration at specific time.

$$
R a_{G}(t)=\frac{d G_{M T}}{d t}-S_{G} G_{b}+\left(S_{G}+S_{I} X_{M T}\right) G_{M T}
$$

in which $G_{M T}$ is plasma glucose concentration in $M T$ and $d G_{M T} / d t$ is the derivative; $X_{M T}$ is the simulated insulin action in MT; $S_{G}, S_{I}$ and $G_{b}$ are parameters. In the present study, because the insulin action $(X)$ can be simulated via the models, Ra of glucose can be calculated directly. The parameter values are obtained in each combination condition, respectively. $d G_{M T} / d t$ is approximated based on the measured plasma glucose concentration at specific times in MT. Cubic splines were used to interpolate both glucose concentration and glucose derivatives with a time step of 1 min consistent with model simulations. Glucose Ra was calculated for each subject, for each Combination, respectively, and then the mean values of calculated $\mathrm{Ra}$ of glucose of each group were further compared with the mean values of model simulated $\mathrm{Ra}$, respectively. The cubic spline used for the glucose concentration and glucose derivative introduces some artifacts, of course, but the results give a rough idea of the consistency of the simulated Ra functional forms with the observed experimental Ra.

\section{Parameter determinations}

The unknown parameters corresponding to each models and each combination (detailed as above), were determined for each subject, respectively.

\section{Objective function}

The unknown parameters were determined for each subject, respectively, based on the following objective function.

$$
\sum_{p} \sum_{m} \sum_{t}\left[\frac{\left(y_{p, m, t}^{\text {Data }}-y_{p, m, t}^{\text {Model }}\right)^{2}}{\sigma_{p, m}^{2}}\right]
$$

where $p \in(\mathrm{IM}-\mathrm{FSIGT}, \mathrm{MT})$ is the experimental protocol; $m \in(\mathrm{G}, \mathrm{F})$ is the measurement of glucose or FFA; $t$ is the data at specific time in each case; $\sigma^{2}$ is the variance, computed as described [30]. Briefly, to compute the variance, we used singular spectrum analysis with only one eigenvalue retained to find a continuous curve that approximated the data for each subject. The standard deviation of the data from this curve provides an estimate of the expected variance of the data from any model fit. The square of this standard deviation per time point is used as $\sigma^{2}$. Thus this variance is a number that is estimated from the raw data, on a per subject basis. It is not optimized so it has no connection to the parameters that are being estimated using the minimization of the cost function. 
Some parameters can be fixed according to experimental measurements. FFA from chylomicron triglyceride does not appear in the plasma until $\sim 60 \mathrm{~min}$. Therefore, in the equation of Ra of FFA, we fixed $t_{O F}=60 \mathrm{~min}$. Moreover, model outputs were found to not be sensitive to some parameters according to our preliminary study, so we fixed $A_{\text {lipo }}=2 ; A_{C l}=2 ; m_{F}=300 \mathrm{~min}$. These simplifications did not influence goodness of fit.

\section{Inputs and outputs}

The inputs of the models are plasma insulin concentration as experimental measurements. The outputs are simulations of plasma glucose and FFA, which are compared with experimental data.

\section{Initial state}

The initial value of insulin action $(X)$ is zero in both IM-FSIGT and MT. The initial condition of glucose or FFA in MT is the measured concentration at the initial time of the MT experiment. In IM-FSIGT, because only data within 10-180 min are considered, the initial value of glucose or FFA for simulation is the average concentration within 0-10 min for glucose or FFA, respectively.

\section{Steady state}

When $X$ vanishes, steady state values are attained as all derivatives in the model equations are zero when insulin, glucose and FFA reach steady state values and Ra vanishes as well.

\section{Model implementation}

The models were implemented in Matlab Version 7.10 (R2010a) (The MathWorks Inc, Natick, MA, http://www.mathworks.com). Parameters were optimized by minimizing the objective function using FMINCON in Matlab. The constraints in optimization are introduced by setting the upper boundaries of parameters, which are chosen based on previous work [30] or a published model [29], typically 5 times the mean values. A change in the upper boundary had minor effects on parameter values. The tolerance is set to $10^{-10}$. The simulations are run on a computer (HP, Z400, Xeon $\left.{ }^{\circ} \mathrm{CPU}, \mathrm{W} 3530,2.8 \mathrm{HGHz}\right)$. In general, the simulation time for one individual is $\sim 5 \mathrm{~s}$ when both IM-FSIGT and MT are considered.

\section{Results}

The present study involves three models (MOD 1, new model; MOD 2 is from Periwal et al.'s study [30]; MOD 3 is from Ramos-Roman et al.'s study [29]). Simulations of each model were performed under four combinations, respectively, dependent on the introduction of glucose/FFA Ra or not and the expression for Ra used: 1) without considering Ra of glucose and FFA; 2) including both glucose and FFA Ra (Type I function); 3) only glucose Ra (Type I function); 4) only glucose Ra (Type II function [40]).

\section{Comparison of models for IM-FSIGT and meal test (MT)}

The average simulated glucose or FFA of each group predicted by each model in IMFSIGT and MT are compared with experimental data in Figs. 1, 2, 3 and 4, corresponding to simulation Combinations 1-4. The fractional residuals in each Combination are shown in Additional file 1: Figures S2-S5. 


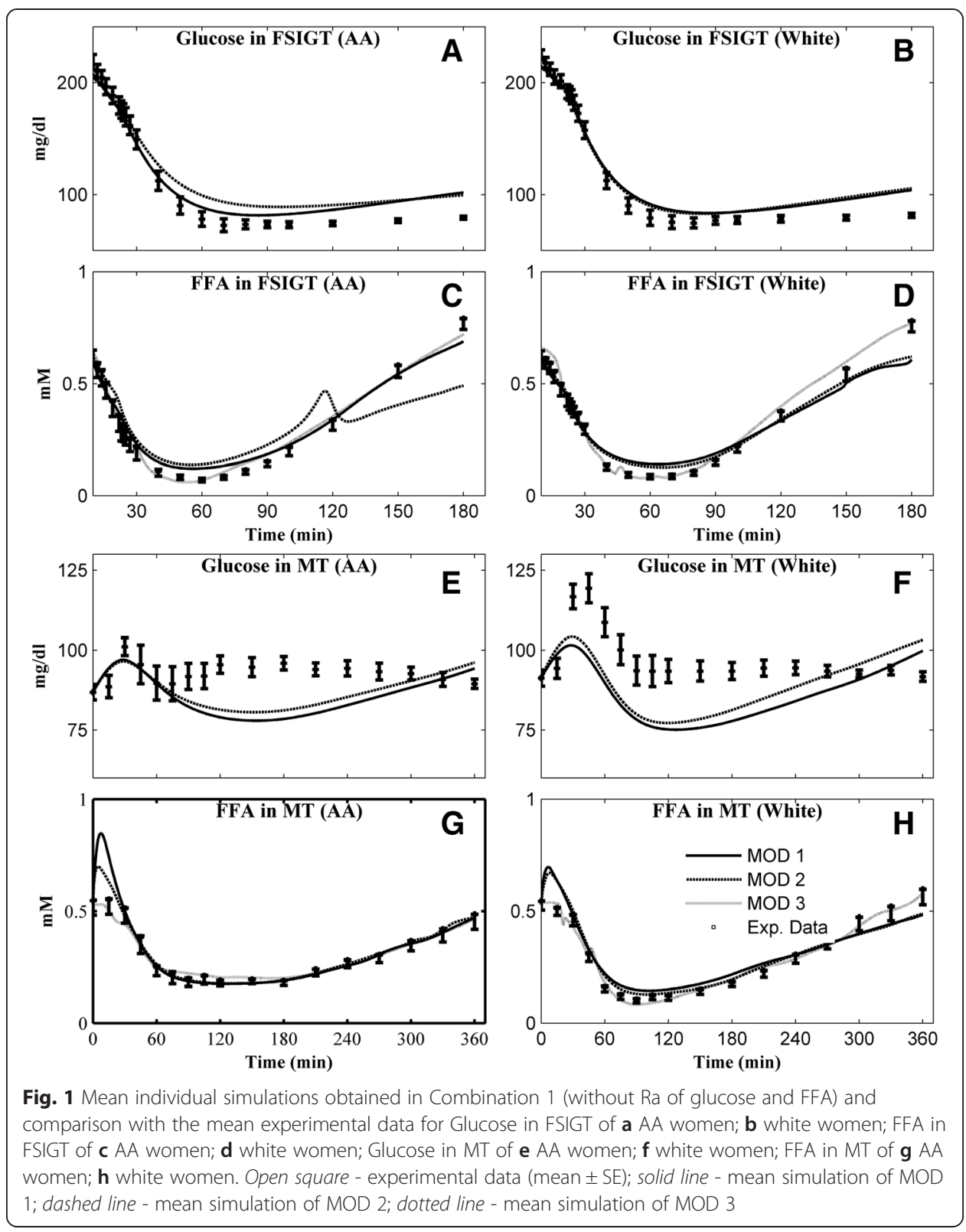

Without introducing Ra (Combination 1), the mean simulated glucose and FFA were compared with experimental data in Fig. 1, and the corresponding mean fraction residuals are shown in Additional file 1: Figure S2. In general, in IMFSIGT, the simulated mean glucose or FFA responses are close to the data for all models (Fig. $1 \mathrm{a}-\mathrm{d}$ ). However, although simulated FFA in MT still matches the data for the models (Fig. $1 \mathrm{~g}, \mathrm{~h}$ ), the simulated glucose deviates from the data (Fig. 1 e, f).

After introducing Ra for both glucose and FFA (Combination 2), the mean predicted glucose and FFA responses of three models are compared with experimental data in Fig. 2. The predicted glucose in IM-FSIGT fit the data well (Fig. $2 \mathrm{a}-\mathrm{b}$ ). The predicted glucose in MT is much closer to data than the simulation in Combination 1 (Fig. 2 e, f) and simulated time-course for white women (Fig. 2 f) matches experimental results 


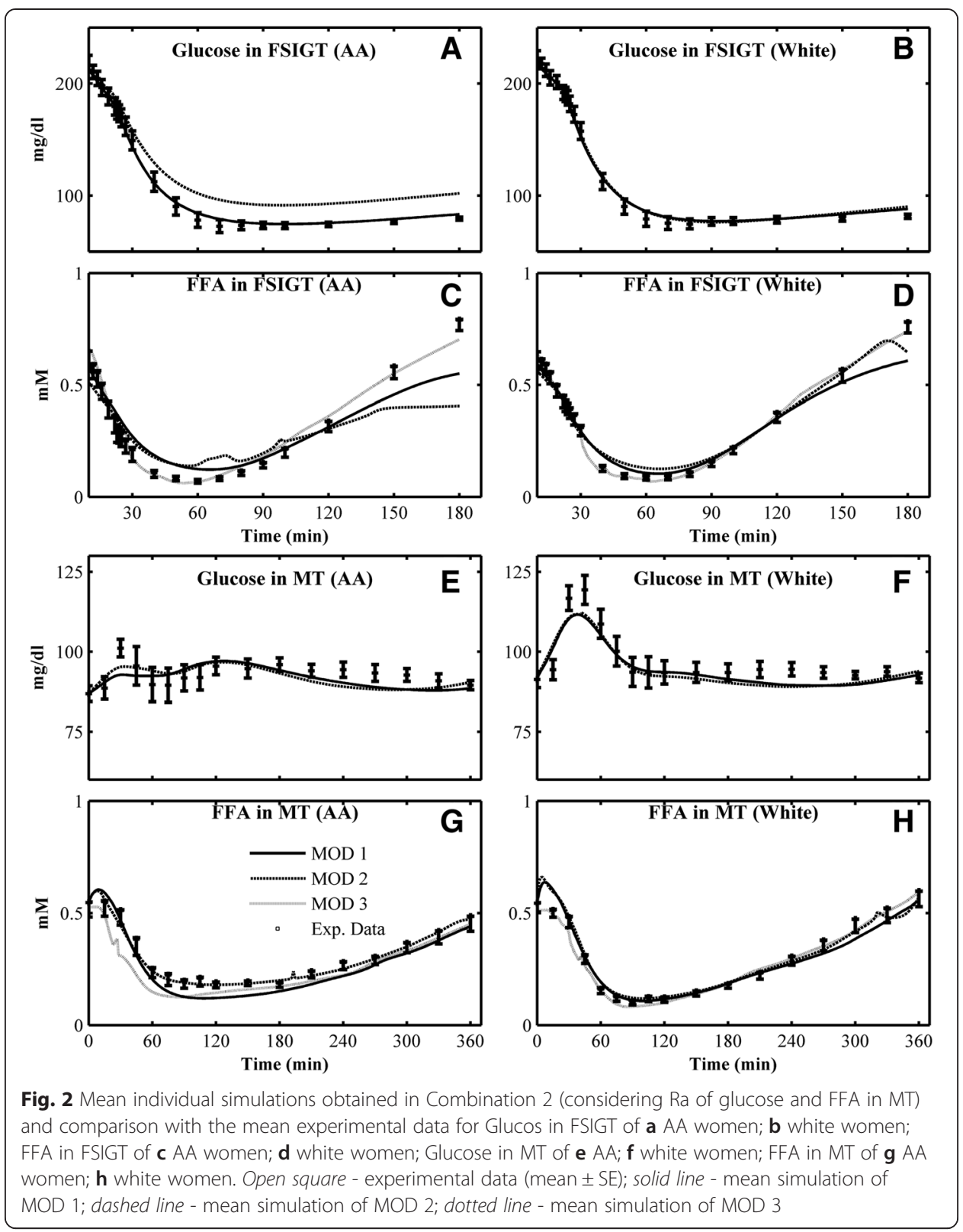

better than for AA women in MT (Fig. 2 e). MOD 3 has better predictive power for FFA data in both tests, FSIGT and MT (Fig. 2 c-d, g-h).

Without considering FFA Ra, the effects of glucose $\mathrm{Ra}$ alone on glucose kinetics are compared in Figs. 3 and 4. Two kinds of glucose Ra functions are considered - Type I (Combination 3) or Type II (Combination 4). Using the Type I function (Combination 3), the mean prediction of glucose and FFA for MOD 1 and MOD 2 are compared with the mean experimental data of AA and white women in Fig. 3. In general, the model predictions of glucose for MOD 1 and MOD 2 are similar to the responses obtained in Combination 2 for both IM-FSIGT and MT. Without Ra of FFA, the fitting of FFA in MT even improved compared to the results from using both glucose and FFA rates of appearance, especially for MOD 2 (Fig. 3 e-f). If the Type II function for the glucose $\mathrm{Ra}$ is used (Combination 4), the mean model prediction of glucose in IM-FSIGT 


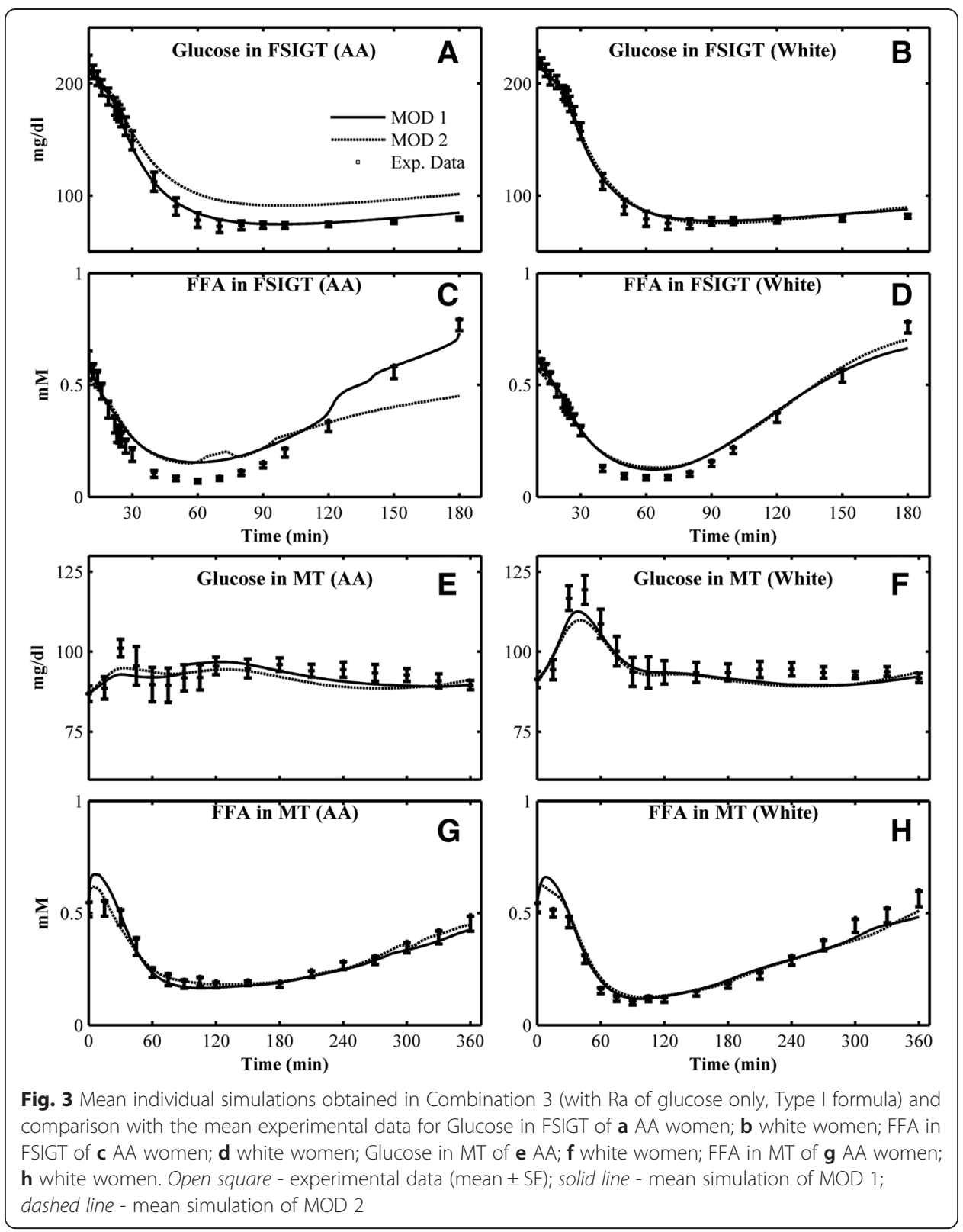

(Fig. $4 \mathrm{a}-\mathrm{b}$ ), and FFA in both of FSIGT and MT (Fig. $4 \mathrm{c}-\mathrm{d}, \mathrm{g}-\mathrm{h}$ ) matches corresponding data. However, the simulated glucose responses in MT (Fig. 4 e-f) deviate from the data for both groups.

The predicted glucose Ra with MOD 1 according to Combinations 2-4 are compared with calculated glucose Ra in Fig. 5. The predicted glucose Ra with the Type I function matches calculated glucose Ra (Fig. 5 a-d) better than simulated glucose Ra with the Type II function (Fig. 5 e-f). This result is consistent with the better fitting with experimental data, which is not only due to more parameters (3 vs. 2), but also different function characteristics (Type II function leads to underestimation of glucose Ra). The abnormal steep drop in the calculated Ra at $\sim 30-60 \mathrm{~min}$ can be attributed to the sparse measurements of glucose in MT, which has dramatic changes during this period and leads to the discontinuous change of glucose change rate (derivative). 


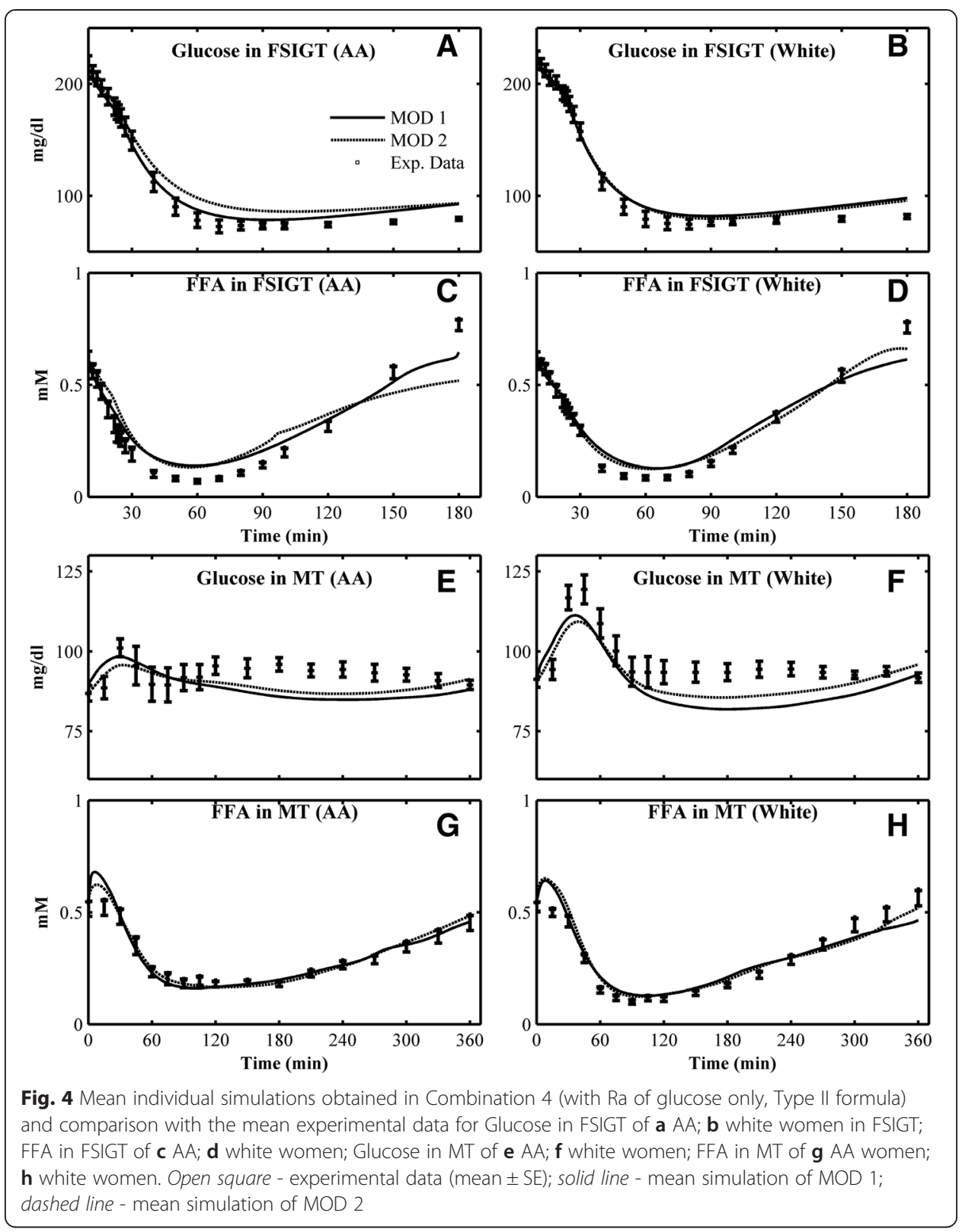

\section{Comparison between groups}

Experimental data of AA and white women in IM-FSIGT and MT are shown in Additional file 1: Figure S1. There are no significant differences in glucose, FFA and insulin kinetics in IM-FSIGT. The parameter values of each group obtained under each Combination with three models are shown in Tables 2, 3 and 4 respectively. In specific cases, several parameters are significantly different between AA and white women, e.g., for MOD 1, white women have larger $S_{I}$ in both Combinations 2 and 3, suggesting more insulin sensitivity (Table 2). The much higher AIRg and DI of AA compared with white women in IM-FSIGT reflects impaired insulin action.

In MT, the elevations of glucose and insulin are smaller than the values in IM-FSIGT (Additional file 1: Figure S1). White women have a higher peak value (at $\sim 45 \mathrm{~min}$ ) than AA women, accompanied by significant higher AUC than AA women within 30-60 min 


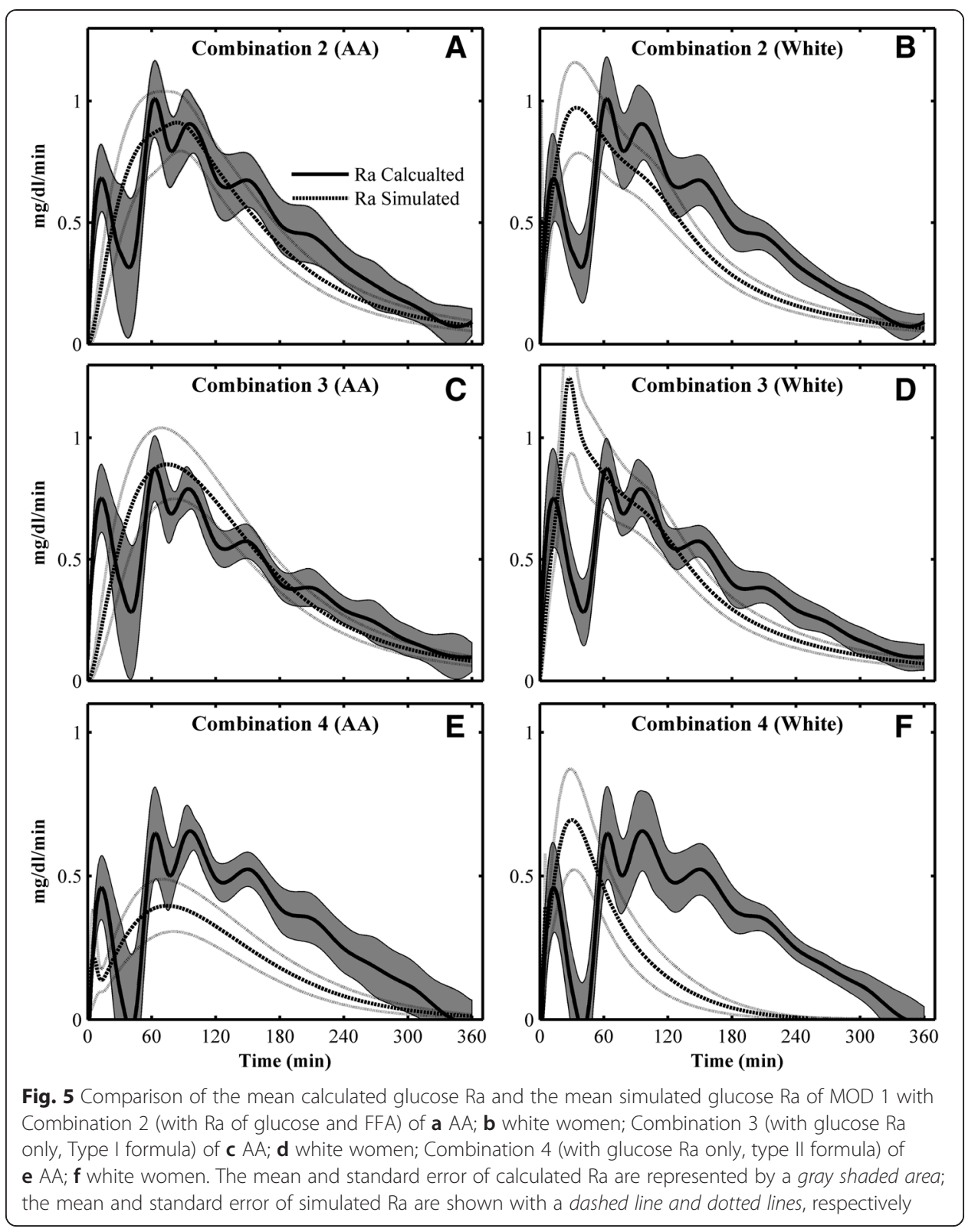

( $p \leq 0.03)$, also reflected in the larger coefficient of glucose $\mathrm{Ra}(\sigma G)$. Predicted Ra of white women also tends to peak earlier than AA women (Fig. 5). These differences suggest that white women may have faster and stronger gastric absorption or specific processes in the liver, or both, which may lead to qualitatively different glucose kinetics - monophasic for white women vs. multiphasic for AA women.

Although the insulin response was relatively smaller in MT compared to IM-FSIGT, the nadir values of FFA in MT were close to the nadir in IM-FSIGT. The relatively smaller insulin regulatory thresholds $\left(X_{2}\right.$ in MOD 1 and MOD 2, $K_{L i p}$ in MOD 3, Tables 2, 3 and 4) than both insulin concentration and simulated insulin action $(X)$ (Additional file 1: Figure S6) suggest that lipolysis in adipose tissue is very sensitive to insulin. In IM-FSIGT, higher insulin levels in AA women accompanied lower FFA levels during 0-60 min (Additional file 1: Figure S1, C, E) compared with the responses 
Table 2 Comparisons of parameters (mean \pm SD) estimated in MOD 1 for each ethnic group in each Combination. Combination 1: without Ra; Combination 2: with Ra for both of glucose and FFA (Type I formula); Combination 3 with Ra of glucose only (Type I formula); Combination 4 with Ra of glucose only (Type II formula). AA: African -American women; white - white women

\begin{tabular}{|c|c|c|c|c|c|c|c|c|}
\hline & \multicolumn{2}{|l|}{ Combination 1} & \multicolumn{2}{|l|}{ Combination 2} & \multicolumn{2}{|l|}{ Combination 3} & \multicolumn{2}{|l|}{ Combination 4} \\
\hline & $\mathrm{AA}$ & White & AA & White & AA & White & AA & White \\
\hline$S_{G}$ & $0.014 \pm 0.007$ & $0.012 \pm 0.008$ & $0.0046 \pm 0.0053$ & $0.0064 \pm 0.0060$ & $5.6 e-3 \pm 5.2 e-3$ & $6.3 e-3 \pm 6.4 e-3$ & $0.012 \pm 0.009$ & $0.0087 \pm 0.0079$ \\
\hline$G_{b}$ & $128.8 \pm 41.1$ & $138.1 \pm 71.2$ & $186.5 \pm 119.9$ & $162.29 \pm 108.2$ & $136.4 \pm 107.9$ & $156.9 \pm 97.8$ & $130.8 \pm 70.1$ & $142.8 \pm 106.7$ \\
\hline$S_{1}$ & $4.1 \mathrm{e}-4 \pm 2.1 \mathrm{e}-4$ & $6.3 e-4 \pm 4.5 e-4$ & $3.1 e-4 \pm 1.5 e-4$ & $5.1 e-4 \pm 3.0 e-4^{a}$ & $3.2 \mathrm{e}-4 \pm 1.4 \mathrm{e}-4$ & $4.9 e-4 \pm 2.6 e-4^{a}$ & $0.0077 \pm 0.026$ & $5.5 e-4 \pm 4.3 e-4$ \\
\hline$C_{x}$ & $0.042 \pm 0.031$ & $0.053 \pm 0.049$ & $0.076 \pm 0.045$ & $0.069 \pm 0.032$ & $0.12 \pm 0.24$ & $0.07 \pm 0.03$ & $0.046 \pm 0.033$ & $0.091 \pm 0.11$ \\
\hline$I_{b x}$ & $5.1 \pm 4.1$ & $5.4 \pm 2.8$ & $3.02 \pm 3.72$ & $4.54 \pm 2.90$ & $4.6 \pm 3.5$ & $4.1 \pm 2.9$ & $5.2 \pm 4.2$ & $5.5 \pm 3.2$ \\
\hline lo & $0.008 \pm 0.01$ & $0.017 \pm 0.038$ & $0.0026 \pm 0.0032$ & $0.003 \pm 0.0028$ & $0.006 \pm 0.007$ & $0.0017 \pm 0.0031^{\mathrm{a}}$ & $0.0086 \pm 0.0092$ & $7.4 e-4 \pm 1.3 e-4^{a}$ \\
\hline$I_{2}$ & $0.14 \pm 0.2$ & $0.12 \pm 0.14$ & $0.12 \pm 0.25$ & $0.048 \pm 0.077$ & $0.12 \pm 0.21$ & $0.052 \pm 0.085$ & $0.089 \pm 0.15$ & $0.049 \pm 0.067$ \\
\hline$x_{2}$ & $14.1 \pm 15.3$ & $14.1 \pm 15.4$ & $12.7 \pm 14.18-$ & $14.1 \pm 15.6$ & $12.7 \pm 15.6$ & $13.6 \pm 15.3$ & $13.1 \pm 15.7$ & $12.6 \pm 14.1$ \\
\hline$C_{f}$ & $0.092 \pm 0.085$ & $0.13 \pm 0.19$ & $0.035 \pm 0.027$ & $0.029 \pm 0.012$ & $0.047 \pm 0.25$ & $0.031 \pm 0.010^{\mathrm{a}}$ & $0.063 \pm 0.044$ & $0.029 \pm 0.012^{a}$ \\
\hline$K_{C l}$ & $25.3 \pm 19.5$ & $15.3 \pm 20.3$ & $23.32 \pm 18.61$ & $12.1 \pm 17.1$ & $21.7 \pm 21.5$ & $7.9 \pm 11.9^{a}$ & $18.6 \pm 16.7$ & $6.1 \pm 13.6^{a}$ \\
\hline$m_{G}$ & - & - & $118.4 \pm 32.96$ & $101.9 \pm 37.1$ & $124.9 \pm 29.7$ & $107.1 \pm 40.7$ & - & - \\
\hline$\sigma G$ & - & - & $0.67 \pm 0.29$ & $1.1 \pm 0.52^{a}$ & $0.66 \pm 0.32$ & $0.90 \pm 0.42$ & - & - \\
\hline$\Delta_{G}$ & - & - & $65.9 \pm 33.6$ & $63.2 \pm 24.3$ & $67.9 \pm 30.6$ & $65.5 \pm 26.6$ & - & \\
\hline$\sigma_{F}$ & - & - & $0.61 \pm 0.38$ & $0.45 \pm 0.31$ & - & - & - & - \\
\hline$\Delta_{F}$ & - & - & $0.32 \pm 0.21$ & $0.36 \pm 0.19$ & - & - & - & - \\
\hline$\varphi_{G}$ & - & - & - & - & & - & $68.3 \pm 41.7$ & $58.1 \pm 40.1$ \\
\hline$\tau_{G}$ & - & - & - & - & - & - & $68.4 \pm 49.1$ & $33.1 \pm 27.8^{a}$ \\
\hline
\end{tabular}


Table 3 Comparisons of parameters (mean \pm SD) estimated in MOD 2 for each ethnic group in each Combination. Combination 1: without Ra of glucose and FFA; Combination 2: with both of Ra of glucose and FFA (Type I formula); Combination 3 with Ra of glucose only (Type I formula); Combination 4 with Ra of glucose only (Type II formula). AA: African -American women; white - white women

\begin{tabular}{|c|c|c|c|c|c|c|c|c|}
\hline & \multicolumn{2}{|l|}{ Combination 1} & \multicolumn{2}{|l|}{ Combination 2} & \multicolumn{2}{|l|}{ Combination 3} & \multicolumn{2}{|l|}{ Combination 4} \\
\hline & $\mathrm{AA}$ & White & $\mathrm{AA}$ & White & $\mathrm{AA}$ & White & $\mathrm{AA}$ & White \\
\hline$S_{G}$ & $0.015 \pm 0.006$ & $0.014 \pm 0.007$ & $0.0061 \pm 0.0057$ & $0.0081 \pm 0.0059$ & $6.5 e-3 \pm 5.6 e-3$ & $7.2 e-3 \pm 46.3 e-3$ & $0.011 \pm 0.0058$ & $8.9 e-3 \pm 7.4 e-3$ \\
\hline$G_{b}$ & $129.5 \pm 25.6$ & $165.9 \pm 63.1$ & $189.7 \pm 92.3$ & $170.54 \pm 79.5$ & $179.3 \pm 84.8$ & $170.5 \pm 74.5$ & $119.9 \pm 45.9$ & $194.2 \pm 103.9^{a}$ \\
\hline$S_{1}$ & $4.1 e-4 \pm 2.3 e-4$ & $5.9 e-4 \pm 2.8 e-4$ & $3.6 e-4 \pm 1.7 e-4$ & $5.9 e-4 \pm 4.7 e-4$ & $3.9 \mathrm{e}-4 \pm 2.0 \mathrm{e}-4$ & $5.5 e-4 \pm 2.6 e-4$ & $4 e-4 \pm 2 e-4$ & $5.8 e-4 \pm 3.9 e-4$ \\
\hline$C_{x}$ & $0.41 \pm 0.029$ & $0.042 \pm 0.023$ & $0.072 \pm 0.071$ & $0.057 \pm 0.029$ & $0.11 \pm 0.19$ & $0.053 \pm 0.019$ & $0.042 \pm 0.024$ & $0.059 \pm 0.038$ \\
\hline$I_{b x}$ & $5.11 \pm 3.82$ & $3.24 \pm 3.23$ & $5.63 \pm 5.33$ & $3.47 \pm 3.99$ & $7.4 \pm 5.6$ & $5.2 \pm 3.8$ & $6.0 \pm 6.1$ & $4.5 \pm 4.2$ \\
\hline lo & $0.0095 \pm 0.015$ & $0.0036 \pm 0.0048$ & $0.033 \pm 0.068$ & $0.0092 \pm 0.024$ & $0.033 \pm 0.061$ & $0.027 \pm 0.082$ & $0.0038 \pm 0.0044$ & $0.023 \pm 0.079$ \\
\hline$I_{2}$ & $0.98 \pm 3.0$ & $0.15 \pm 0.21$ & $0.24 \pm 0.28$ & $0.17 \pm 0.27$ & $0.23 \pm 0.32$ & $0.19 \pm 0.33$ & $0.13 \pm 0.2$ & $0.14 \pm 0.25$ \\
\hline$x_{2}$ & $11.7 \pm 12.3$ & $12.8 \pm 15.8$ & $12.12 \pm 14.81$ & $12.9 \pm 14.8$ & $13.4 \pm 15.4$ & $13.2 \pm 15.9$ & $15.6 \pm 16.8$ & $13.1 \pm 16.0$ \\
\hline$A_{l}$ & $2.19 \pm 1.01$ & $2.57 \pm 0.77$ & $2.13 \pm 1.13$ & $2.45 \pm 0.82$ & $2.1 \pm 0.91$ & $2.4 \pm 0.8$ & $2.3 \pm 0.99$ & $2.4 \pm 0.79$ \\
\hline$C_{f O}$ & $0.15 \pm 0.16$ & $0.19 \pm 0.28$ & $0.25 \pm 0.34$ & $0.17 \pm 0.24$ & $0.23 \pm 0.32$ & $0.22 \pm 0.35$ & $0.092 \pm 0.044$ & $0.13 \pm 0.24$ \\
\hline$m_{G}$ & - & - & $122.31 \pm 30.13$ & $100.9 \pm 44.9$ & $124.6 \pm 31.1$ & $99.4 \pm 44.9$ & - & - \\
\hline$\sigma G$ & - & - & $0.66 \pm 0.41$ & $0.93 \pm 0.65$ & $0.71 \pm 0.43$ & $0.78 \pm 0.4$ & - & - \\
\hline$\Delta_{G}$ & - & - & $62.7 \pm 33.2$ & $59.6 \pm 29.7$ & $61.2 \pm 32.6$ & $59.3 \pm 27.9$ & - & - \\
\hline$\sigma_{F}$ & - & - & $0.60 \pm 0.38$ & $0.28 \pm 0.29^{a}$ & - & - & - & - \\
\hline$\Delta_{f}$ & - & - & $0.53 \pm 0.39$ & $0.68 \pm 0.34$ & - & - & - & - \\
\hline$\varphi_{G}$ & - & & - & - & - & - & $77.2 \pm 37.9$ & $83.7 \pm 22.1$ \\
\hline$\tau_{G}$ & - & & - & - & - & - & $82.9 \pm 44.0$ & $64.2 \pm 39.4$ \\
\hline
\end{tabular}

a: indicate significantly different between AA and white women in each Combination 
Table 4 Comparisons of model parameters of each ethnic group in each Combination with MOD 3. Combination 1: without Ra of FFA. Combination 2: with Ra term of FFA (Type I formula, because MOD 3 only includes FFA kinetics, so only Combinations 1 and 2 are involved). AA: African American women; White: White women

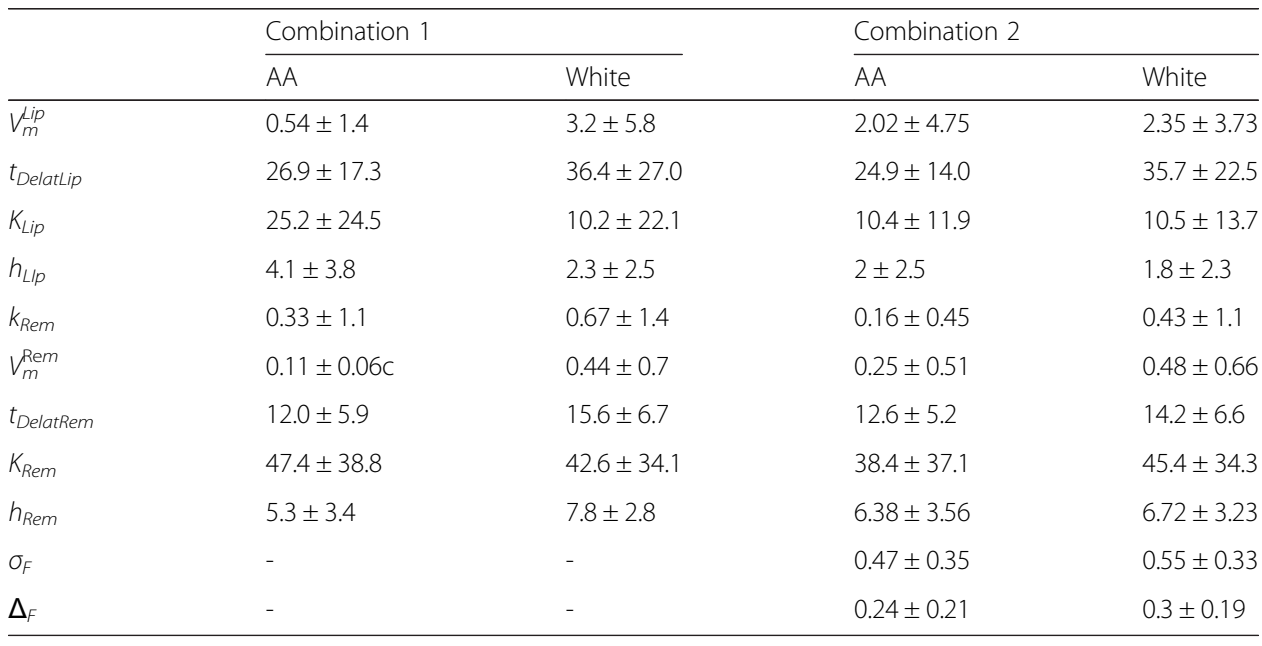

of white women. However, in MT, although AA women still had higher insulin, FFA levels are significantly higher than white women during 60-180 $\min (p \leq 0.04)$. This reversal could, perhaps, be attributed to a different modulation of lipolysis inhibition. In fact, white women tend to reach the nadir faster than AA women $(\sim 80 \mathrm{~min}$ vs. $130 \mathrm{~min})$. This suggests that the inhibition of lipolysis for AA women may have differences in insulin signaling in adipose tissue.

Predictions for insulin action kinetics $(X)$ in IM-FSIGT or MT are compared with insulin data in Additional file 1: Figure S6. The kinetics of $X$ in MT are closer to the measured insulin (Additional file 1: Figure S6 C, D). In both IM-FSIGT and MT, the responses of $X$ appear to have a time delay compared with insulin, which is consistent with the experimental observations [12].

\section{Model comparison}

Those models under four Combinations are compared with the Bayes information criterion (BIC) in Table 5. Clearly, when both glucose and FFA data are involved, MOD 1 produces a better fit than MOD 2, specifically for Combination 3 (Type I function for

Table 5 Model comparison using Bayes information criterion (BIC) ${ }^{a}$

\begin{tabular}{lllr}
\hline Model & Combination & AA & \multicolumn{1}{c}{ White } \\
\hline MOD 1 & 1 & $361.9 \pm 81.7$ & $338.9 \pm 62.9$ \\
& 2 & $345.7 \pm 93.1$ & $335.7 \pm 69.8$ \\
& 3 & $320.5 \pm 95.5$ & $307.1 \pm 67.5$ \\
& 4 & $328.4 \pm 87.8$ & $316.8 \pm 64.1$ \\
MOD 2 & 1 & $437.4 \pm 84.7$ & $382 \pm 56.9$ \\
& 2 & $420.7 \pm 83.3$ & $360.7 \pm 78.4$ \\
& 3 & $401.1 \pm 74.5$ & $337.51 \pm 76.6$ \\
& 4 & $405.2 \pm 84.0$ & $343.6 \pm 61.6$ \\
\hline
\end{tabular}

a: MOD 3 only covers FFA kinetics, so it cannot be compared with MOD 1 and MOD 2 directly 
glucose Ra, no FFA Ra). The root mean square error (RMSE) of each model and each Combination are shown in Table 6, also verifying upon results. The results obtained based on addition combinations (S1-S3) show that there is no significant difference for the parameters between two ethnic groups in those specific conditions, only FSIGT (S1) or only MT (S2, S3).

\section{Discussion}

The primary aims of this study were to define a simple function to estimate the appearance rate ( $\mathrm{Ra})$ of glucose and FFA in MT and to refine an existing model of FFA and glucose kinetics and test its suitability for modeling post-prandial FFA and glucose. As an application, we demonstrated how the contributions of Ra of glucose or FFA on the corresponding kinetics could be quantitatively evaluated. Our new model provides a practical approach to evaluate glucose kinetics in MT.

\section{Effect of Ra on glucose and FFA kinetics}

As expected, we found that glucose Ra plays an essential role in glucose kinetics in MT. Without glucose Ra (Combination 1), the predicted glucose responses completely fail to match the data (Fig. 1 e-f). This is not a surprise, of course, but even the form of the glucose Ra matters: the model predictions based on the Type II function were not satisfactory (Fig. 4, e-f). The fit for white women (Figs. 2 and 3, f) was better than AA women (Figs. 2 and 3, e). Our results suggest that for individuals with monophasic glucose responses in MT, a simple function (Type I, similar to a log-normal functional form) can be used to model glucose Ra in MT (Figs. 2, 3f), making it easier to evaluate glucose kinetics with fewer parameters.

In contrast, for all three models, the simulations with or without introduction of FFA Ra had relatively small differences (Fig. 1 g-h, vs. Figs. 2, 3 and 4, g-h), suggesting that the effect of FFA Ra on post-prandial FFA in MT is limited. This in consistent with studies showing that dietary FFA will not appear in plasma until after $\sim 2 \mathrm{~h}$ [22]. Because FFA responses in IM-FSIGT and MT are quite similar, lipolysis and clearance

Table 6 Model comparison of goodness of fit. The value is the root mean square error (RMSE). In the calculation, the residual is normalized by the variance of raw data

\begin{tabular}{|c|c|c|c|c|c|c|}
\hline \multirow[t]{2}{*}{ Combination } & \multirow[t]{2}{*}{ Description } & \multirow[t]{2}{*}{ Model } & \multicolumn{2}{|c|}{ Glucose } & \multicolumn{2}{|l|}{ FFA } \\
\hline & & & FSIGT & MT & FSIGT & MT \\
\hline \multirow[t]{3}{*}{1} & \multirow[t]{3}{*}{ No Ra } & 1 & 226.1 & 254.2 & 306.4 & 137.9 \\
\hline & & 2 & 284.1 & 214.2 & 860.2 & 138.7 \\
\hline & & 3 & - & - & 264.7 & 122.5 \\
\hline \multirow[t]{3}{*}{2} & \multirow[t]{3}{*}{ Both Ra (Type I) } & 1 & 179.2 & 104.1 & 378.6 & 146.8 \\
\hline & & 2 & 326.3 & 114.3 & 471.9 & 165.1 \\
\hline & & 3 & - & - & 289.6 & 121.9 \\
\hline \multirow[t]{3}{*}{3} & \multirow[t]{2}{*}{ Only Ra glucose } & 1 & 182.5 & 100.1 & 239.3 & 135.5 \\
\hline & & 2 & 298.8 & 119.4 & 527.8 & 165.4 \\
\hline & (Type I) & 3 & - & - & - & - \\
\hline \multirow[t]{3}{*}{4} & \multirow[t]{3}{*}{ Only Ra glucose (Type II) } & 1 & 208.6 & 203.2 & 219.7 & 129.5 \\
\hline & & 2 & 266.5 & 149.6 & 846.1 & 148.8 \\
\hline & & 3 & - & - & - & - \\
\hline
\end{tabular}


between FSIGT and MT should have similar magnitudes, in agreement with model predictions (Additional file 1: Figure S7, A vs. C, B vs. D). Model simulated FFA Ra did not reach comparable levels with lipolysis and clearance until after 300 min (Additional file 1: Figure S7, C-D). The Ra of FFA can therefore be neglected in modeling postprandial kinetics.

\section{Comparison of model behaviors}

Model performance is dependent on both of goodness-of-fit and model complexity. BIC was used to compare models in various Combinations, in which MOD 1 under Combination 3 (with Type I glucose Ra) is better than others. Although MOD 3 has better fits for FFA, it only cover FFA data and cannot be directly compared with MOD 1 and MOD 2. The comparison of root mean square error (Table 6) under each condition of three models also reflects those results.

In MOD 3, plasma insulin is applied directly with a specific time delay for lipolysis or clearance. This approach has been utilized in other models as well [17]. However, for peripheral tissues, the effects of insulin on glucose or FFA are generally dependent on the activation of insulin signaling, which then impacts other processes, e.g., the translocation of GLUT4 in skeletal muscle. Because of distinct time courses and magnitudes of insulin signaling and insulin concentration, it may not be physiologically accurate to use the insulin concentration directly, even with a time delay, as suggested by experimental study [12].

The kinetics of the disappearance rate of glucose are different from insulin kinetics [41], suggesting that insulin acts through a insulin action $(X)$ as used in MOD 1 and MOD 2. According to a previous study [30], the assumption that glucose and FFA are related to different remote insulin actions did not improve the model while adding complexity.

Mathematical studies have attempted to estimate dynamic glucose $\operatorname{Ra}[23,27]$. Especially, experimental measured glucose $\mathrm{Ra}$ showed that glucose $\mathrm{Ra}$ is similar to a log-normal function [38]. In the present study, two functions of glucose Ra are compared (Type I and Type II [40]). Both of them can simulate responses similar to the expected $\mathrm{Ra}$ profile of glucose. The two functions have 3 or 2 free parameters, respectively. The results of Type I are much better than the simulations of Type II (Combination 3 vs. Combination 4 for both MOD 1 and MOD 2 (Figs. 2, 3 and 4, e-f), as evident in the improved BIC value that prefers this model. Therefore, with only 3 parameters, the Type I equation is a good choice to estimate glucose Ra in the postprandial Combination for a monophasic glucose responses, compared with the complicated mathematical calculations in some studies [23, 27].

\section{Approaches to model simplification}

In the lipid uptake term, the insulin-independent clearance constant and the maximal clearance rate coefficient were combined together as one parameter $\left(C_{f}\right.$, Eq. B6 in Additional file 1). Because FFA is a mixture of fatty acids of different chain lengths, the short and middle chain FFA can enter into peripheral tissue by simple diffusion. Insulin only influences long chain FFA [33, 35, 37, 42]. Our definition balances the roles of different FFA and had little effect on model predictions. The Ra of glucose is simpler than previous studies [25-27]. 


\section{Model limitations and future improvement}

In agreement with the literature [43], glucose of AA women had multiphasic characteristics (Additional file 1: Figure S1, B). Although simulations using the Type I function work well for white women, but they do not fit data for AA women. Therefore Type I glucose Ra may be inappropriate for specific individuals. Either a better functional form of glucose Ra for multiphasic cases needs to be developed, or a distinct model of glucose-insulin dynamics for MT versus FSIGT which is consistent with the same monophasic Ra of glucose needs to be developed. Clearly, a tracer study would be the appropriate way to test which possibility correctly represents the ethnicity-dependent MT and FSIGT kinetics. The reasons for more complex glucose kinetics in AA women in MT need further study. Predictions of MOD 1 still partly deviate from FFA data in the lipolysis inhibition phase, during which FFA drops more rapidly than predicted by the model. This may be due to parasympathetic activity triggered by the meal.

With the data available, we were not able to address the second limitation of the MM approach (mentioned in the Introduction) in this work, but we note that the meal test MM we developed here may be a better starting point towards an autonomic MM. Such an autonomic system would have parameters for insulin secretion as a function of glucose, but almost certainly would implicitly include a quantification of glucagon dynamics and hepatic glucose output. This is an active area of research and no consensus has appeared to our knowledge.

\section{Conclusion}

In conclusion, we developed a single MM for glucose and FFA that is appropriate for both IM-FSIGT and MT. Data from both IM-FSIGT and MT can be fitted well with this model. The model (MOD 1) attempts to balance complexity and goodness of fit while keeping the mechanisms modeled physiologically plausible. Model simulations show that white women may have higher insulin sensitivity. The Ra of FFA in MT may not play an important role in FFA kinetics. The expression for glucose Ra that we developed, which is similar to a log-normal functional form, provides a way to simplify the estimation of glucose dynamics in MT in some cases.

\section{Additional file}

Additional file 1: Supplemental materials. (DOC $386 \mathrm{~kb}$ )

Competing interests

The authors declare that they have no competing interests.

\section{Authors' contributions}

CCC, PV and LY developed the models; LY performed the model simulations; LY and PV wrote the manuscript; CAB and CCC edited the manuscript; SAE provided clinical research data and edited the manuscript; CAB administered the clinical protocol. All authors read and approved the final manuscript.

\section{Acknowledgements}

Li, Chow, Sumner and Periwal were supported by the Intramural Research Program of the National Institutes of Health, NIDDK and Courville was supported by the Clinical Center of the NIH.

\section{Author details}

'Laboratory of Biological Modeling, National Institute of Diabetes and Digestive and Kidney Diseases (NIDDK), National Institutes of Health (NIH), MSC 5621, LBM, NIDDK, NIH, Bethesda, MD 20892-5621, USA. ${ }^{2}$ Nutrition Department, Clinical Center, National Institute of Diabetes and Digestive and Kidney Diseases (NIDDK), National Institutes of Health (NIH), Bethesda, MD 20892, USA. ${ }^{3}$ Diabetes, Endocrinology, and Obesity Branch, National Institute of Diabetes and Digestive and Kidney Diseases (NIDDK), National Institutes of Health (NIH), Bethesda, MD 20892, USA. 
Received: 11 July 2015 Accepted: 26 February 2016

Published online: 02 March 2016

\section{References}

1. Arner P. Human fat cell lipolysis: biochemistry, regulation and clinical role. Best Pract Res Clin Endocrinol Metab. 2005; 19:471-82.

2. $\quad$ Arner P, Ryden M. Fatty Acids, Obesity and Insulin Resistance. Obes Facts. 2015;8:147-55.

3. Arner P. Free fatty acids - do they play a central role in type 2 diabetes? Diabetes Obes Metab. 2001;3 Suppl 1:11-9.

4. $\quad$ Arner P. Insulin resistance in type 2 diabetes: role of fatty acids. Diabetes Metab Res Rev. 2002;18 Suppl 2:S5-9.

5. Adler-Wailes DC, Periwal V, Ali AH, Brady SM, McDuffie JR, Uwaifo Gl, et al. Sex-associated differences in free fatty acid flux of obese adolescents. J Clin Endocrinol Metab. 2013;98:1676-84.

6. Sumner AE, Micklesfield LK, Ricks M, Tambay AV, Avila NA, Thomas F, et al. Waist circumference, BMI, and visceral adipose tissue in white women and women of African descent. Obesity (Silver Spring). 2011;19:671-4.

7. Chow CC, Periwal V, Csako G, Ricks M, Courville AB, Miller 3rd BV, et al. Higher acute insulin response to glucose may determine greater free fatty acid clearance in African-American women. J Clin Endocrinol Metab. 2011;96:2456-63.

8. Singh B, Saxena A. Surrogate markers of insulin resistance: A review. World J Diabetes. 2010;1:36-47.

9. Katz A, Nambi SS, Mather K, Baron AD, Follmann DA, Sullivan G, et al. Quantitative insulin sensitivity check index: a simple, accurate method for assessing insulin sensitivity in humans. J Clin Endocrinol Metab. 2000;85:2402-10.

10. Cobelli C, Man CD, Sparacino G, Magni L, De Nicolao G, Kovatchev BP. Diabetes: Models, Signals, and Control. IEEE Rev Biomed Eng. 2009;2:54-96.

11. Bergman RN, Ider YZ, Bowden CR, Cobelli C. Quantitative estimation of insulin sensitivity. Am J Physiol. 1979;236:E667-77.

12. Bergman RN. Lilly lecture 1989. Toward physiological understanding of glucose tolerance. Minimal-model approach. Diabetes. 1989;38:1512-27.

13. Caumo A, Vicini P, Zachwieja JJ, Avogaro A, Yarasheski K, Bier DM, et al. Undermodeling affects minimal model indexes: insights from a two-compartment model. Am J Physiol. 1999;276:E1171-93.

14. Bergman RN. Minimal model: perspective from 2005. Horm Res. 2005;64 Suppl 3:8-15.

15. Palumbo P, Ditlevsen S, Bertuzzi A, De Gaetano A. Mathematical modeling of the glucose-insulin system: a review. Math Biosci. 2013;244:69-81.

16. Erion DM, Shulman Gl. Diacylglycerol-mediated insulin resistance. Nat Med. 2010;16:400-2.

17. Boston RC, Moate PJ. A novel minimal model to describe NEFA kinetics following an intravenous glucose challenge. Am J Physiol Regul Integr Comp Physiol. 2008;294:R1140-7.

18. Morbiducci U, Di Benedetto G, Kautzky-Willer A, Deriu MA, Pacini G, Tura A. Identification of a model of non-esterified fatty acids dynamics through genetic algorithms: the case of women with a history of gestational diabetes. Comput Biol Med. 2011;41:146-53.

19. Roy A, Parker RS. Dynamic modeling of free fatty acid, glucose, and insulin: an extended "minimal model". Diabetes Technol Ther. 2006;8:617-26.

20. Lambert JE, Parks EJ. Postprandial metabolism of meal triglyceride in humans. Biochim Biophys Acta. 2012;1821:721-6.

21. Zierler K. Whole body glucose metabolism. Am J Physiol. 1999;276:E409-26.

22. Fielding B. Tracing the fate of dietary fatty acids: metabolic studies of postprandial lipaemia in human subjects. Proc Nutr Soc. 2011;70:342-50.

23. Dalla Man C, Caumo A, Basu R, Rizza R, Toffolo G, Cobelli C. Minimal model estimation of glucose absorption and insulin sensitivity from oral test: validation with a tracer method. Am J Physiol Endocrinol Metab. 2004;287:E637-43.

24. Gillis R, Palerm CC, Zisser H, Jovanovic L, Seborg DE, Doyle FJ. Glucose estimation and prediction through meal responses using ambulatory subject data for advisory mode model predictive control. J Diabetes Sci Technol. 2007; 1:825-33.

25. Herrero P, Bondia J, Palerm CC, Vehi J, Georgiou P, Oliver N, et al. A simple robust method for estimating the glucose rate of appearance from mixed meals. J Diabetes Sci Technol. 2012;6:153-62.

26. Hovorka R, Jayatillake H, Rogatsky E, Tomuta V, Hovorka T, Stein DT. Calculating glucose fluxes during meal tolerance test: a new computational approach. Am J Physiol Endocrinol Metab. 2007;293:E610-9.

27. Dalla Man C, Caumo A, Cobelli C. The oral glucose minimal model: estimation of insulin sensitivity from a meal test. IEEE Trans Biomed Eng. 2002;49:419-29.

28. Jelic K, Hallgreen CE, Colding-Jorgensen M. A model of NEFA dynamics with focus on the postprandial state. Ann Biomed Eng. 2009;37:1897-909.

29. Ramos-Roman MA, Lapidot SA, Phair RD, Parks EJ. Insulin activation of plasma nonesterified fatty acid uptake in metabolic syndrome. Arterioscler Thromb Vasc Biol. 2012;32:1799-808.

30. Periwal V, Chow CC, Bergman RN, Ricks M, Vega GL, Sumner AE. Evaluation of quantitative models of the effect of insulin on lipolysis and glucose disposal. Am J Physiol Regul Integr Comp Physiol. 2008;295:R1089-96.

31. Heiling VJ, Jensen MD. Free fatty acid metabolism in the follicular and luteal phases of the menstrual cycle. J Clin Endocrinol Metab. 1992;74:806-10

32. Sumner AE, Furtado JD, Courville AB, Ricks M, Younger-Coleman N, Tulloch-Reid MK, et al. ApoC-III and visceral adipose tissue contribute to paradoxically normal triglyceride levels in insulin-resistant African-American women. Nutr Metab (Lond). 2013;10:73.

33. Bonen A, Chabowski A, Luiken JJ, Glatz JF. Is membrane transport of FFA mediated by lipid, protein, or both? Mechanisms and regulation of protein-mediated cellular fatty acid uptake: molecular, biochemical, and physiological evidence. Physiology (Bethesda). 2007;22:15-29.

34. Glatz JF, Luiken JJ, Bonen A. Membrane fatty acid transporters as regulators of lipid metabolism: implications for metabolic disease. Physiol Rev. 2010;90:367-417. 
35. Kazantzis M, Stahl A. Fatty acid transport proteins, implications in physiology and disease. Biochim Biophys Acta. 2012;1821:852-7.

36. Ramos-Roman MA, Sweetman L, Valdez MJ, Parks EJ. Postprandial changes in plasma acylcarnitine concentrations as markers of fatty acid flux in overweight and obesity. Metabolism. 2012;61:202-12.

37. Schwenk RW, Holloway GP, Luiken JJ, Bonen A, Glatz JF. Fatty acid transport across the cell membrane: regulation by fatty acid transporters. Prostaglandins Leukot Essent Fatty Acids. 2010;82:149-54.

38. Pennant ME, Bluck LJ, Marcovecchio ML, Salgin B, Hovorka R, Dunger DB. Insulin administration and rate of glucose appearance in people with type 1 diabetes. Diabetes Care. 2008;31:2183-7.

39. Roust $L R$, Jensen MD. Postprandial free fatty acid kinetics are abnormal in upper body obesity. Diabetes. 1993;42: 1567-73.

40. Pearson T, Wattis JA, King JR, MacDonald IA, Mazzatti DJ. A mathematical model of the human metabolic system and metabolic flexibility. Bull Math Biol. 2014;76:2091-121.

41. Jacobsen SH, Bojsen-Moller KN, Dirksen C, Jorgensen NB, Clausen TR, Wulff BS, et al. Effects of gastric bypass surgery on glucose absorption and metabolism during a mixed meal in glucose-tolerant individuals. Diabetologia. 2013;56:2250-4.

42. Wu Q, Ortegon AM, Tsang B, Doege H, Feingold KR, Stahl A. FATP1 is an insulin-sensitive fatty acid transporter involved in diet-induced obesity. Mol Cell Biol. 2006;26:3455-67.

43. Tura A, Morbiducci U, Sbrignadello S, Winhofer Y, Pacini G, Kautzky-Willer A. Shape of glucose, insulin, C-peptide curves during a 3-h oral glucose tolerance test: any relationship with the degree of glucose tolerance? Am J Physiol Regul Integr Comp Physiol. 2011;300:R941-8.

\section{Submit your next manuscript to BioMed Central and we will help you at every step:}

- We accept pre-submission inquiries

- Our selector tool helps you to find the most relevant journal

- We provide round the clock customer support

- Convenient online submission

- Thorough peer review

- Inclusion in PubMed and all major indexing services

- Maximum visibility for your research

Submit your manuscript at www.biomedcentral.com/submit 